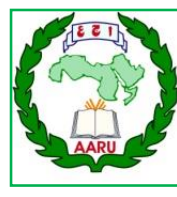

Arab Univ. J. Agric. Sci., Ain Shams Univ., Cairo, Egypt 29(2), 667-676, 2021

Website: http://ajs.journals.ekb.eg

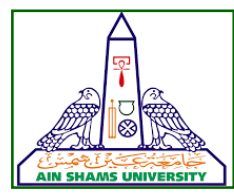

DOI: 10.21608/ajs.2021.67476.1348

\title{
A Comparative Study Between Different Preservation Methods on The Viability of Some Yeast Cultures
}

\section{Reham KM Abdallah*, Ibrahim SA Rizk, Amal AM Hassan, Hemat E Elsheshetawy}

Food Sciences Dept, Fac of Agric, Ain Shams Univ, P.O. Box 68, Hadayek Shoubra 11241, Cairo, Egypt

*Corresponding author: RehamAbdallah2015@agr.asu.edu.eg

\begin{abstract}
The influence of chilling, freezing, and drying preservation methods on morphological properties and viability of four yeast cultures, isolated Saccharomyces boulardii (SB1 and $\mathrm{SB} 2$ ), Saccharomyces cerevisiae (SC), and Kluyveromyces lactis (KL), was studied. chilling preservation had no effect on yeast colony morphology parameters till the end of the preservation period in contrast with freezing and drying preservation methods. Drying preservation resulted in the highest viability decrement $\%$ of yeast cultures ranged between 59.38 to $40.21 \%$ as compared with other preservation methods. There is a noticeable reduction in $D$ values of preserved yeast cultures by drying, which varied from (33 to 26 day) in comparison to chilling and freezing preservation that recorded (119 to 83 and 180 to 57 day), respectively. The obtained results revealed that chilling preservation under proper conditions, as well as freezing, is more preferable for the preservation of studied yeast cultures than that preserved yeast by the drying method.
\end{abstract}

Keywords: yeast, preservation, morphology, viability, $\mathrm{D}$ value

\section{Introduction}

Yeasts have served as a workhorse for both research and industrial applications due to their genetic and biochemical malleability. Microbial cultivation and characterization alone are not adequate without proper preservation methods to maintain microbes (Kulkarni and Chitte 2015). Thus, appropriate preservation methods are required to enhancing long-term availability of microbial cells without loss in their metabolic potentials. The basic principle of microbial preservation methods is a reduction of microbial metabolism without disturbing cell integrity (Jain et al 2020). Preservation of yeasts is very important because they are used as starter cultures in the manufacturing of bakery products, alcoholic beverages, and other sectors.

Among several methods devised for preserving yeast cultures, periodic subculture on agar slants or plates is the most common one (Ravimannan 2016). The agar slant method can be divided into butt and slant/inclined portion were serving as a reservoir of nutrients. On refrigeration, the metabolic activities of cells reduce due to low temperature, but the activities do not get arrested. Desiccation preservation method is successful for yeast survival on filter paper although it may be unsuitable for other genera (Bassel et al 1977). Agar slant and desiccation methods are simple steps and 
not require expensive apparatus. They are suitable for laboratories with limited resources and occasion with high ambient temperature regions.

Freeze-drying, liquid-drying, and freezing are various methods for long-term preservation of microorganisms (Tan and Mustapha 2014). Freezing yeast gave the ability to preserve and build large collections which promote scientific and industrial innovations (Cabrera et al 2020).

This research aimed to compare between different preservation methods by evaluating the viability of studied yeasts after preservation. The best method to be employed was determined according to proper viability and preservation time.

\section{Material and Methods}

Yeast growth medium (YPD) was obtained from HiMedia, Mumbai, India. Saccharomyces boulardii (SB1) perocur ( $S$. cerevisiae HANSEN CBS 5926) was purchased from Hexal AG company, Poland. Saccharomyces boulardii - 90 DRcaps $®$ (SB2) was purchased from Fairvital Company, German. Saccharomyces cerevisiae ATCC 64712 (SC) and Kluyveromyces lactis CBS 2359 (KL) were obtained from MERCEN, Ain Shams University, Cairo, Egypt.

The two Saccharomyces boulardii strains were isolated and identified by PCR amplification and rDNA sequencing of the internal transcribed spacer region (ITS) in Sigma laboratory, 6 October, Egypt (Barros Lopes et al 1996). The obtained database was compared with the BLAST search (http://www.ncbi.nlm.nih.gov/BLAST/, NCBI, Bethesda, MD, USA). All yeast cultures were tested for their morphological characteristics (Kurtzman and Fell 1998). Also, the turbidity of grown yeast cultures was determined by spectrophotometer at $600 \mathrm{~nm}$ (Mensah and Tagoe 2018). The cultures were subjected to three preservation methods. Firstly, chilling preservation was adopted according to (Ravimannan 2016) where agar slants of YPD were inoculated in triplicate for each yeast culture and stored at $5 \pm 1^{\circ} \mathrm{C}$ (Kirshop and Snell 1984). Secondly, freezing preservation method was described by (Tan and Mustapha 2014). Sterilized cryoprotective medium (glycerol 50 $\%, \mathrm{v} / \mathrm{v}$ ) was added to yeast cultures and mixed gently to make a suspension which transferred to cryotubes and stored in a freezer at -20 $\pm 1^{\circ} \mathrm{C}$. Chilled and frozen yeast cultures were checked periodically through 150 days for viability by using the spread plate method. Thirdly, drying preservation was applied on yeast cultures using the desiccation method by McCartney bottles according to ( Karbaum 1989). Dried cultures were counted for viability periodically during 105 days of storage.

\section{Statistical analysis}

Differences in viable yeast numbers were statistically analyzed using T-test at a degree of significance $(p \leq 0.05)$. It was performed for measuring specific differences between means by using the SAS, V9 software system.

\section{Results and Discussion}

\subsection{Identification of yeast cultures and Phylogenetic Analysis}

The main target of yeast isolation and identification from commercial Saccharomyces boulardii in this study is to assure that isolated yeasts are already Saccharomyces boulardii cultures and at the same time, the two isolated strains are different from each other. After determining the sequence of rDNA of the ITS region for two isolated yeasts a database search for similar sequences in the GenBank was performed using the BLASTn program. Fig 1. represented the phylogenetic trees and taxonomy lineage of isolated Saccharomyces boulardii SB1 $(\mathrm{a}, \mathrm{b})$ and SB2 $(\mathrm{a}, \mathrm{b})$ based on homology of $16 \mathrm{~S}$ rDNA sequencing of ITS. As shown in Fig 1, SB1a and SB2a, a phylogenetic tree was mapped using the neighbor-joining method, and the sequences of isolated yeasts were found to be similar to strains belonging to the genus Saccharomyces. Also, Fig 


\section{A Comparative Study Between Different Preservation Methods on The Viability of Some Yeast Cultures}

1., SB1b and SB2b illustrate the taxonomy lineage of SB1 and SB2 isolates. This Figure showed that the SB1 strain was similar to Saccharomyces boulardii with a 769 score and one hit where the isolated SB2 took a 1055 score with 4 hits. These results mean that the isolated cultures had a strong relationship with Saccharomyces $\mathrm{sp}$. As mentioned by (Valente et al 1996), yeasts belonging to Saccharomyces sp. had a shorter ITS region, so this characteristic is most useful in the identification of Saccharomyces isolates.

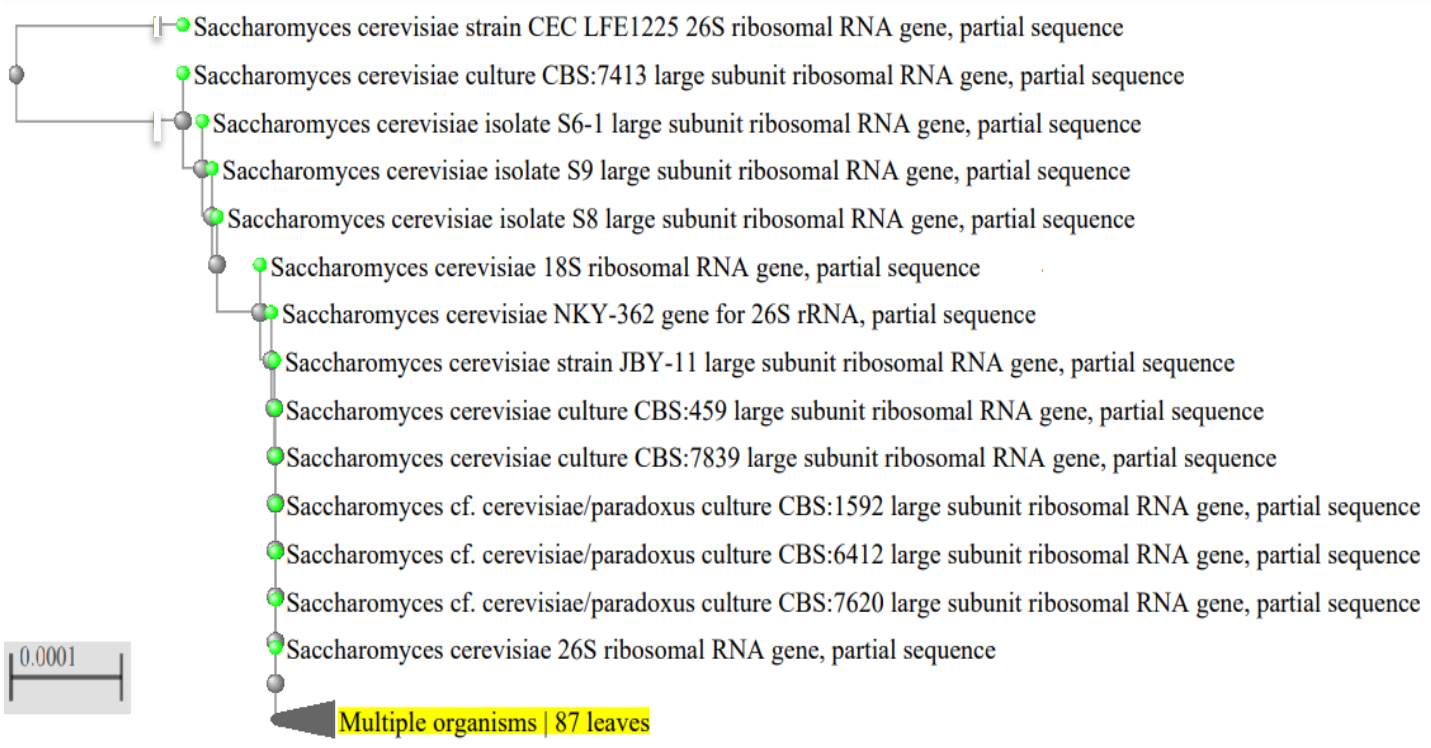

SB1a

\begin{tabular}{|c|c|c|c|c|c|c|}
\hline \multirow{3}{*}{$\begin{array}{l}\text { Descriptions } \\
\text { Reports }\end{array}$} & & & & & & \\
\hline & \multicolumn{2}{|c|}{ Graphic Summary } & Alignments & Taxonomy & lomy & \\
\hline & Lineage & Organism & Taxonomy & & & \\
\hline \multicolumn{7}{|c|}{100 sequences selected } \\
\hline \multicolumn{3}{|c|}{ Organism } & Blast Name & Score & Number of Hits & Des \\
\hline \multicolumn{3}{|c|}{ Fungi } & \multicolumn{2}{|l|}{ fungi } & $\underline{100}$ & \\
\hline \multicolumn{3}{|c|}{ - Saccharomycetales } & \multicolumn{2}{|l|}{$\underline{\text { budding.yeasts }}$} & $\underline{99}$ & \\
\hline \multicolumn{3}{|c|}{. Saccharomyces } & \multicolumn{2}{|l|}{ budding.yeasts } & $\underline{98}$ & \\
\hline \multicolumn{3}{|c|}{. S Saccharomyces cerevisiae } & budding.yeasts & 778 & $\underline{92}$ & $\underline{\text { Saccharomyces cerevisiae hits }}$ \\
\hline \multicolumn{3}{|c|}{. . Saccharomyces cf. cerevisiae/paradoxus } & $\underline{\text { budding.yeasts }}$ & 773 & $\underline{4}$ & $\underline{\text { Saccharomyces cf. cerevisiae/par }}$ \\
\hline \multicolumn{3}{|c|}{. . Saccharomyces boulardii (nom inval.) } & $\underline{\text { budding.yeasts }}$ & 769 & 1 & Saccharomyces boulardii (nom in \\
\hline \multicolumn{3}{|c|}{. . Saccharomyces sp. } & budding.yeasts & 769 & 1 & $\underline{\text { Saccharomyces sp. hits }}$ \\
\hline \multicolumn{3}{|c|}{. Pichia kudriavzevii } & budding.yeasts & 767 & 1 & Pichia kudriavzevii hits \\
\hline \multicolumn{3}{|c|}{ - uncultured yeast } & fungi & 769 & 1 & uncultured yeast hits \\
\hline
\end{tabular}

SB1b 


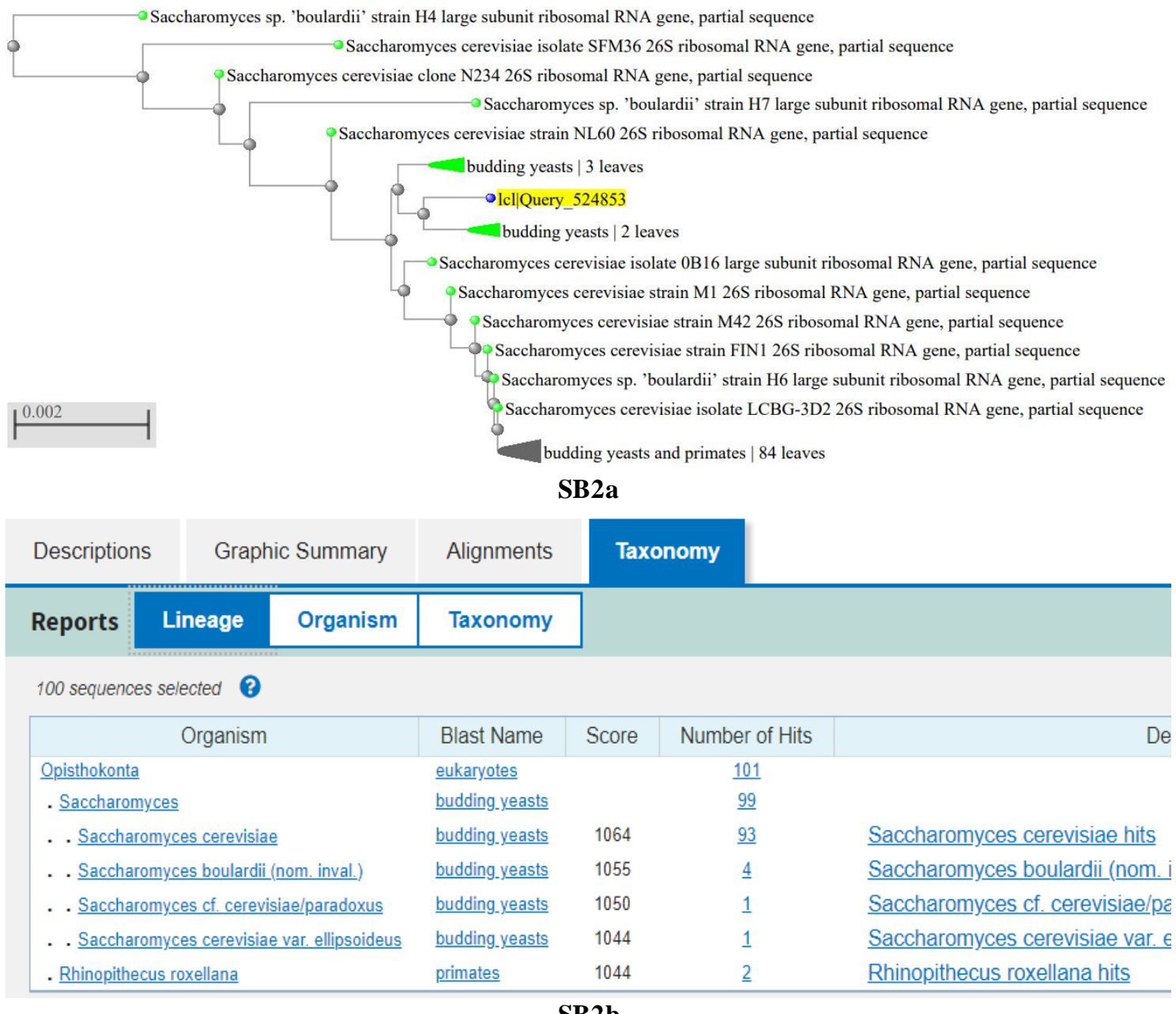

Fig 1. Phylogenetic trees and taxonomy lineage of isolated Saccharomyces boulardii SB1 (a,b) and SB2 $(a, b)$ based on homology of $16 \mathrm{~S}$ rDNA sequencing of ITS.

\subsection{Effect of preservation methods on mor- phological characteristics of yeast cultures}

Morphological characteristics of yeast colonies on the YPD solid medium were illustrated in Table 1 and Fig 2. The studied yeast cultures were tested for color, colony morphology parameters (elevation, shape, and texture), colony size, and turbidity. Colonies of fresh yeast cultures (SB1, SB2, and SC) have a cream color, where colonies of KL culture have a white color. These results are in accordance with those of (Montes et al 2016), who referred to the yeast cells are characterized as flat, smooth, moist, glistening, or dull, with cream to tannish cream color. (Widiastutik and Alami 2013), as well as (Suriasih et al 2012) report that the studied yeast was white to creamy butyrous texture, raised, convex surface, opaque and ovoid in shape. After preservation, yeast color was not affected by preservation methods as shown in Table 1 and Fig 2. Colony morphology parameters were not affected by chilling preservation, where freezing preservation changed the elevation of SC colonies from raised to flat and drying preservation changed the elevation of SB1, SB2, and $\mathrm{SC}$ colonies from raised to flat also as shown in Fig 2. Yeast colony size was affected by the preservation method as presented in Table $\mathbf{1}$. 
A Comparative Study Between Different Preservation Methods on The Viability of Some Yeast Cultures

Table 1 Morphological characteristics of different yeast cultures as affected by preservation methods

\begin{tabular}{|c|c|c|c|c|}
\hline Strains & Color & Colony morphology & $\begin{array}{c}\text { Colony size } \\
\text { decrement \% }\end{array}$ & $\begin{array}{c}\text { Turbidity } \\
\text { decrement } \%\end{array}$ \\
\hline & \multicolumn{4}{|c|}{ 1- Fresh cultures } \\
\hline SB 1 & Creamy & Raised, Ovoid, Smooth & 0 & 0 \\
\hline SB2 & Creamy & Raised, Circular, Smooth & 0 & 0 \\
\hline $\mathrm{SC}$ & Creamy & Raised, Circular, Smooth & 0 & 0 \\
\hline \multirow[t]{2}{*}{ KL } & White & Flat, Circular, Smooth & 0 & 0 \\
\hline & \multicolumn{4}{|c|}{ 2- Cultures afterchilled storage } \\
\hline SB 1 & Creamy & Raised, Ovoid, Smooth & 23.2 & 0.42 \\
\hline SB2 & Creamy & Raised, Circular, Smooth & 14.6 & 0.49 \\
\hline $\mathrm{SC}$ & Creamy & Raised, Circular, Smooth & 12.1 & 1.66 \\
\hline \multirow[t]{2}{*}{ KL } & White & Flat, Circular, Smooth & 7.7 & 0.97 \\
\hline & \multicolumn{4}{|c|}{ 3- Cultures after freezing storage } \\
\hline SB 1 & Creamy & Raised, Ovoid, Smooth & 39.2 & 36.85 \\
\hline SB2 & Creamy & Raised, Circular, Smooth & 38.0 & 7.68 \\
\hline $\mathrm{SC}$ & Creamy & Flat, Circular, Smooth & 34.0 & 2.03 \\
\hline \multirow[t]{2}{*}{ KL } & White & Flat, Circular, Smooth & 30.9 & 70.61 \\
\hline & \multicolumn{4}{|c|}{ 4- Cultures after drying storage } \\
\hline SB 1 & Creamy & Flat, Ovoid, Smooth & 52.5 & 82.25 \\
\hline SB2 & Creamy & Flat, Circular, Smooth & 48.9 & 80.37 \\
\hline $\mathrm{SC}$ & Creamy & Flat, Circular, Smooth & 46.1 & 81.70 \\
\hline KL & White & Flat, Circular, Smooth & 44.5 & 84.40 \\
\hline
\end{tabular}

S. boulardii (SB1) \& (SB2), Saccharomyces cerevisiae (SC) and Kluyveromyces lactis (KL)

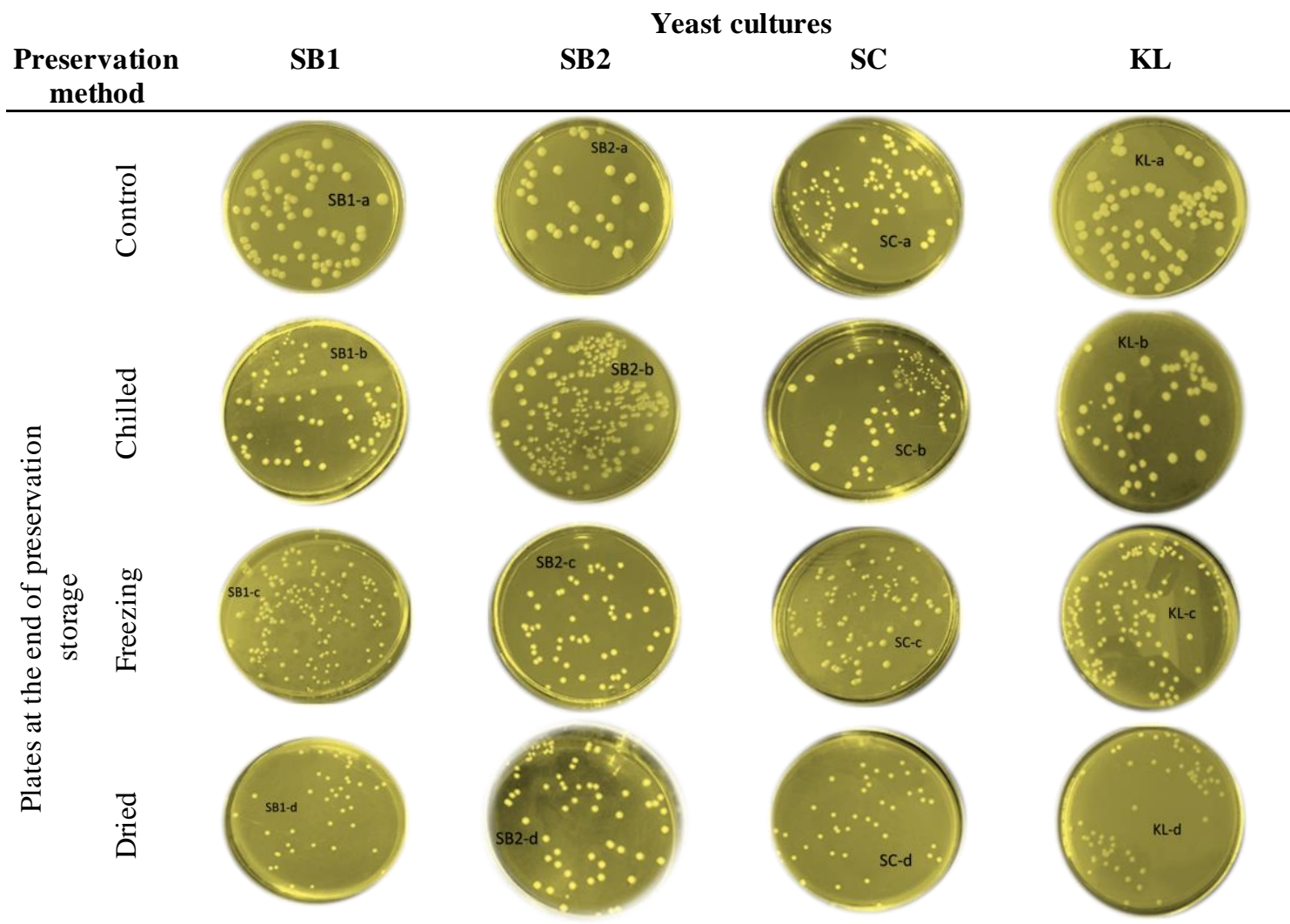

Fig 2. Morphology of yeast colonies on YPD plates under different preservation methods S. boulardii (SB1) \& (SB2), Saccharomyces cerevisiae (SC) and Kluyveromyces lactis (KL) 
The highest colony size decrement $\%$ was achieved by SB1 culture after drying storage whereas the lowest one was achieved by KL culture after chilled storage. The turbidity of growing yeast was affected by preservation methods. The lowest adverse effect was achieved by chilling preservation which reached $1.66 \%$ for SC culture as can be seen in Table 1. With respect to turbidity, data revealed that freezing preservation weakens all yeast cultures, where the most affected yeast strain was KL which recorded the highest percentage of decrement $(70.61 \%)$. In contrast, the drying preservation affect strongly on the turbidity of all growing yeast cultures with a decrement $\%$ reached $84.40 \%$ for KL culture. The obtained results showed that chilling preservation is considered the best method since it maintained the morphological characteristics of the preserved yeast cultures.

\subsection{Effect of preservation methods on viabil- ity of yeast cultures}

Four different yeast cultures (SB1, SB2, $\mathrm{SC}$, and $\mathrm{KL}$ ) were preserved under chilling and freezing conditions for 150 days and under drying conditions for 105 days. All different yeast cultures significantly decreased $(\mathrm{p} \leq$ 0.05 ) in the checked viable count during the storage period under all preservation methods. $\mathrm{D}$ value, which denotes the decimal reduction time, is a time required for reduction of microbial population by one decimal at specific temperature and conditions. D values of preserved yeast cultures were calculated from linear relations between viable count and storage time under different preservation methods as shown in Figs 3, 4 and 5 and Table 2. At chilling preservation, the $\mathrm{D}$ values ranged from the highest value 119 to the lowest value 83 day for SB1 and KL, respectively. Under freezing conditions, $\mathrm{D}$ values ranged from the highest value 180 for SB2 to the lowest value 57 day for SB1 and SC cultures. It was observed that the lowest $\mathrm{D}$ values were obtained by the drying preservation method. $D$ values of dried cultures ranged from 33 to 26 day for SB2 and $\mathrm{SC}$, respectively. Dried preservation decreased the $D$ values forall yeast cultures notably when compared with chilling and freezing preservation methods. Viability decrement $\%$ was calculated for all preserved yeast cultures. The results of decrement $\%$ are consistent with the obtained D values for all yeast cultures. Although the dried preservation had the lowest $\mathrm{D}$ values and the highest viability decrement $\%$. However, the injury values for yeast cultures preserved by drying and chilling were almost identical. From the linear relation between the viable count and the storage period, it can be deduced the predicted death time when the yeast cultures will lose their viability. The predicted death time was ranged from approximately 2.8 to 1.9 year for SB 1 and KL cultures, respectively, under chilled storage. This time was ranged from 4 to 1.2 year for SB2 and SC, respectively, under freezing storage while it was ranged from 0.8 to 0.5 year for SB2 and SC, respectively, under drying storage. The obtained results are in the same trend with those of (Grover et al 1967, Bridge 1966), they referred to the viability loss of certain microbes when stored at $-20{ }^{\circ} \mathrm{C}$, which may be due to the damages of the cells. Also, they added that at the storage range of $-10^{\circ}$ to -40 ${ }^{\circ} \mathrm{C}$, most of the cells contents may not be frozen fully, and the concentration of electrolytes becomes very high in the unf rozen parts. (Kawamura et al 1995, Qiangqiang et al 1998, Crespo et al 2000, Mariano et al 2003) stated that freezing and freeze-drying of strains are very useful for long-term storage and are commonly employed for the preservation of a variety of microorganisms, including yeast and microorganisms with special requirements. (Antheunisse et al 1981) mentioned that the yeast cells recovery on dried filter paper after the storage is much lower than that of the cells kept on sealed agar slants or in lyophilized conditions. 

on The Viability of Some Yeast Cultures

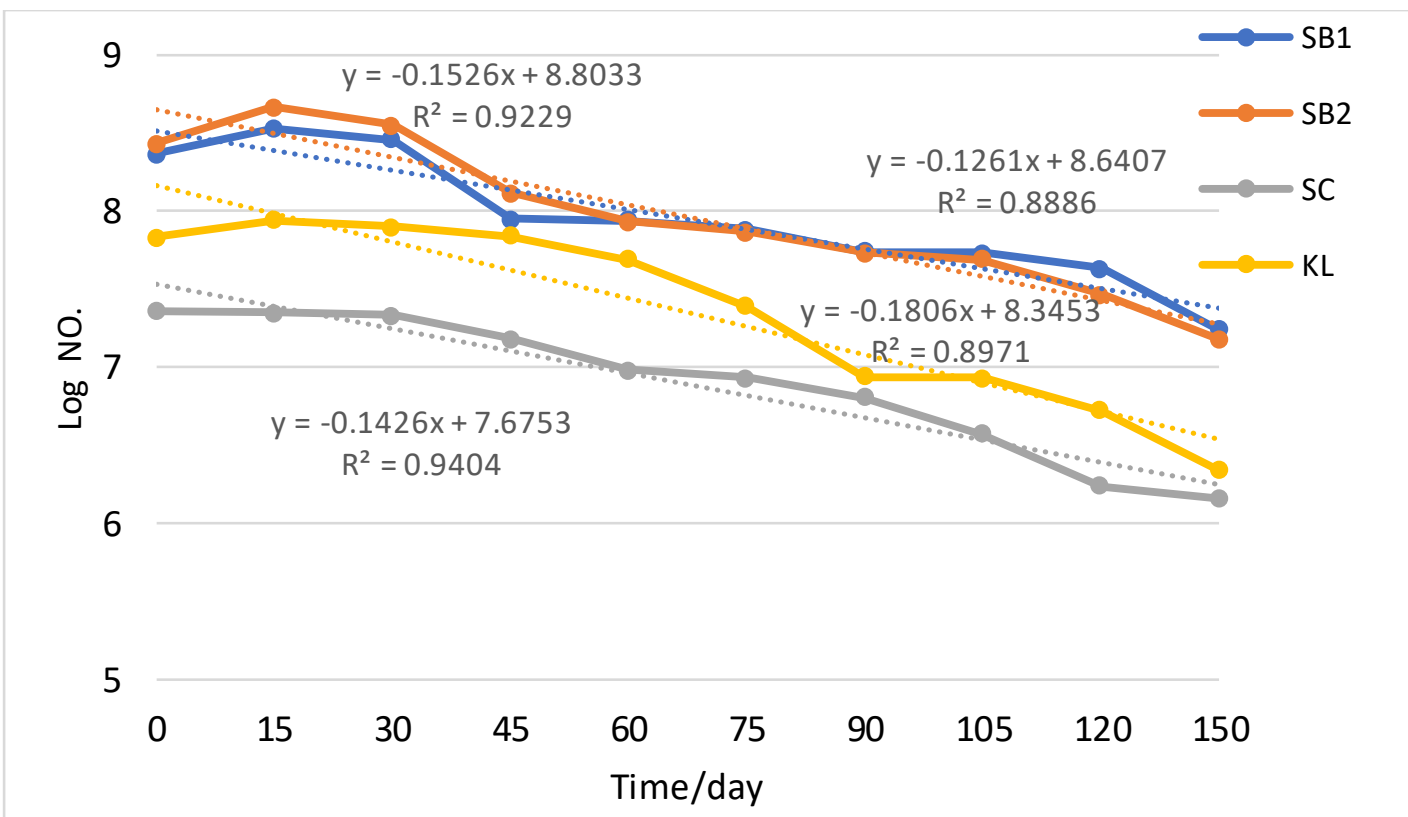

Fig 3. Effect of chilling preeservation on viability of yeast cultures

S. boulardii (SB1) \& (SB2), Saccharomyces cerevisiae (SC) and Kluyveromyces lactis (KL)

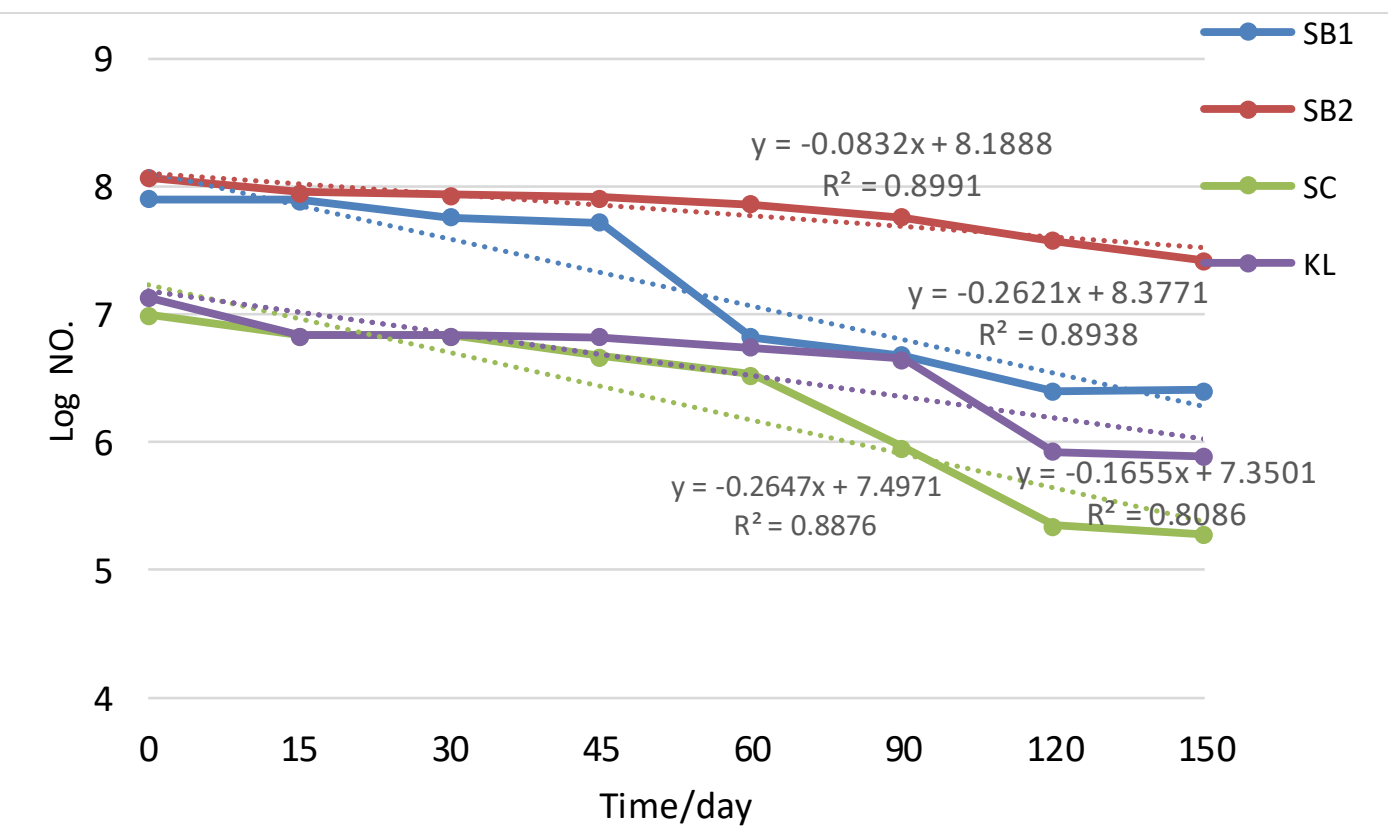

Fig 4. Effect of freezing preservatin on viabilty of yeast culture

S. boulardii (SB1) \& (SB2), Saccharomyces cerevisiae (SC) and Kluyveromyces lactis (KL) 


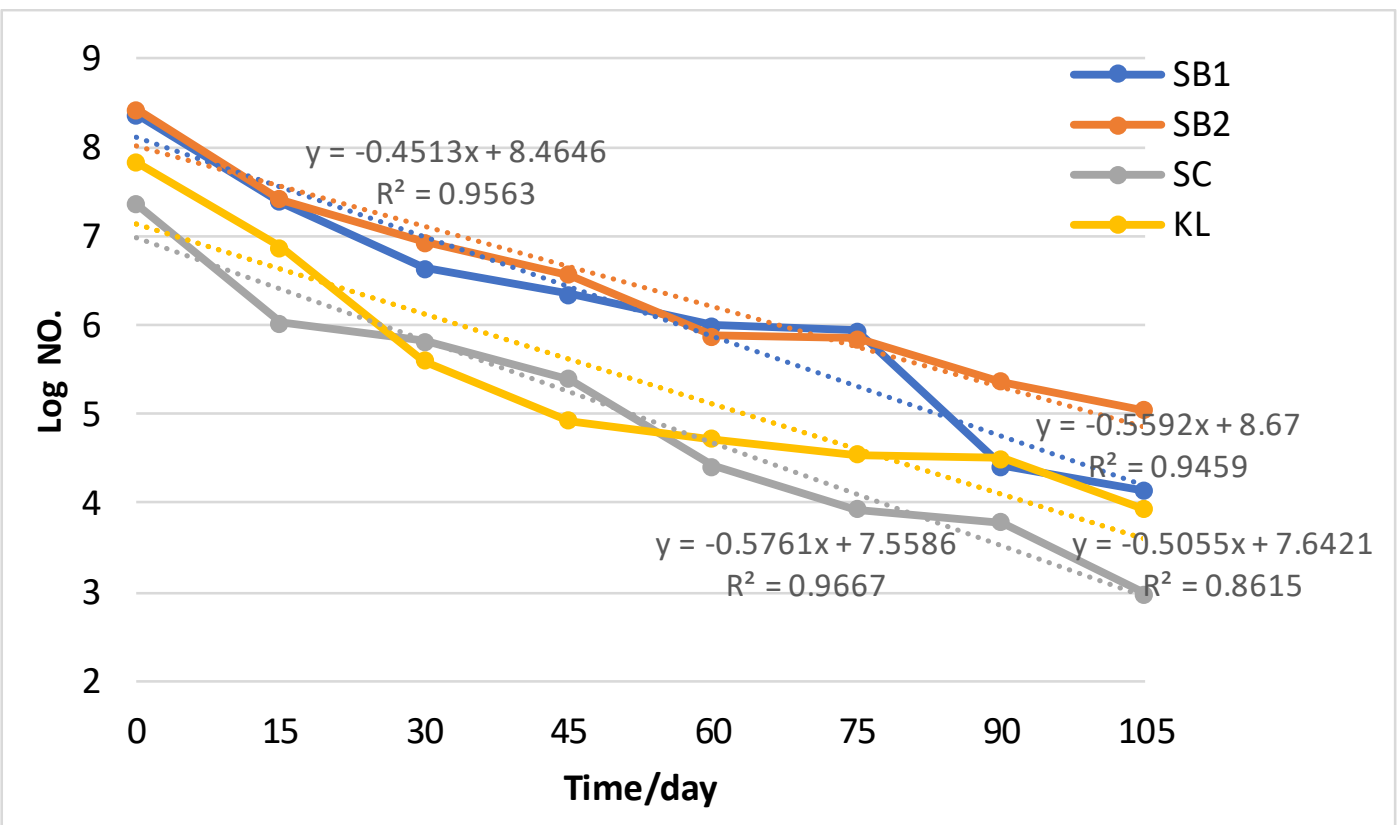

Fig 5. Effect of drying preservation on viability of yeast cultures

S. boulardii (SB1) \& (SB2), Saccharomyces cerevisiae (SC) and Kluyveromyces lactis (KL)

Table 2. D values, viable decrement and injury cell \% of different yeast cultures under different preservation methods

\begin{tabular}{|c|c|c|c|c|c|c|c|c|c|}
\hline \multirow{3}{*}{$\begin{array}{c}\text { Yeast } \\
\text { cultures }\end{array}$} & \multicolumn{3}{|c|}{ Chilling } & \multicolumn{3}{c|}{ Freezing } & \multicolumn{3}{c|}{ Drying } \\
\cline { 2 - 11 } & $\begin{array}{c}\text { D value/ } \\
\text { day }\end{array}$ & $\begin{array}{c}\text { Viability } \\
\text { decrement } \\
\%\end{array}$ & $\begin{array}{c}\text { Injury } \\
\%\end{array}$ & $\begin{array}{c}\text { D value/ } \\
\text { day } \\
\text { Viability } \\
\text { decrement } \\
\%\end{array}$ & $\begin{array}{c}\text { Injury } \\
\%\end{array}$ & $\begin{array}{c}\text { D value/ } \\
\text { day }\end{array}$ & $\begin{array}{c}\text { Viability } \\
\text { decrement } \\
\%\end{array}$ & $\begin{array}{c}\text { Injury } \\
\%\end{array}$ \\
\hline SB1 & 119 & 13.5 & 0.83 & 57 & 18.86 & 7.33 & 27 & 50.54 & 0.72 \\
\hline SB2 & 98 & 14.83 & 0.70 & 180 & 8.02 & 5.66 & 33 & 40.21 & 0.79 \\
\hline SC & 105 & 16.3 & 0.32 & 57 & 24.57 & 6.44 & 26 & 59.38 & 1.00 \\
\hline KL & 83 & 19.03 & 0.47 & 91 & 17.43 & 1.87 & 30 & 49.81 & 0.76 \\
\hline
\end{tabular}

S. boulardii (SB1) \& (SB2), Saccharomyces cerevisiae (SC) and Kluyveromyces lactis (KL)

\section{Conclusion}

This study reports the effect of three preservation methods, chilling, freezing, and drying, on the viability of four yeast cultures. It was shown that according to the obtained results, chilling preservation under proper conditions as well as freezing pre servation is more preferable for the preservation of studied yeast cultures than that by the drying preservation method. 


\section{A Comparative Study Between Different Preservation Methods on The Viability of Some Yeast Cultures}

\section{References}

Antheunisse J, de Bruin-Tol JW, van der PolVan Soest ME (1981) Survival of microorganisms after drying and storage. J. Antonie van Leeuwenhoek 47, 539-545.

DOI:10.1007/BF00443240

Barros Lopes M, Soden A, Henschke PA, Langridge P (1996) PCR differentiation of commercial yeast strains using intron splice site primers. Appl Environ Microbiol 62, 45144520.

Bassel J, Contopoulou R, Mortimer R, Fogel S (1977) In UK Federation for Culture Collections Newsletter 4, 1- 7.

Bridges BA (1966) Preservation of microorganisms at low temperatures. Lab Pract 15, 418-422.

Cabrera E, Welch LC, Robinson MR, Sturgeon CM, Crow MM, Segarra VA (2020) Cryopreservation and the Freeze-Thaw Stress Response in Yeast, review. J Genes 11, 1-11. https://www.mdpi.com/2073-4425/11/8/835

Crespo MJ, Abarca ML, Cabañes FJ (2000) Evaluation of different preservation and storage methods for Malassezia spp. J. Clin. Microbiol 38, 3872-3875.

Grover AA, Kim HK, Wiegeshaus EH, Smith DW (1967) Host-parasite relationships in experimental airborne tuberculosis II. Reproducible infection by means of an inoculum preserved at $-70^{\circ} \mathrm{C}$. J Bacteriol $94,832-835$.

Jain A, Jain R, Jain S (2020). Basic Techniques in Biochemistry, Microbiology and Molecular Biology. Springer Protocols Handbooks. ISBN 978-1-4939-9861-6 (eBook), pp 2354. https://doi.org/10.1007/978-1-4939-9861-6

Kawamura Y, Hou X, Sultana F, Miura H, Ezaki T (1995) Determination of 16s rRNA Sequences of Streptococcus mitis and Streptococcus gordonii and Phylogenetic Relationships among Members of the Genus Streptococcus. Int J of Systematic Bacteriology 45, 406-408.
Kirshop BE, Snell JJS (1984) Maintenance of microorganisms: a manual of laboratory methods. Academic Press pp.207.

Karbaum K (1989) Living resources for biotechnology: Filamentous fungi. Cambridge, New York, New Rochelle, Melbourne, Sydney: Cambridge University Press pp 209. https://doi.org/10.1002/abio.370090503

Kulkarni GA, Chitte RR (2015) Preservation of thermophilic bacterial spores using filter paper disc techniques. J Bioprocessing \& Biotechniques 5, 223. DOI:10.4172/2155$\underline{9821.1000223}$

Kurtzman CP, Fell JW (1998) In: The Yeasts: A Taxonomic Study (Eds.: Kurtzman, C.P., Fell, J.W.). Elsevier, Amsterdam, pp 2354.

Mariano PLS, Milan EP, da Matta DA, Colombo AL (2003) Candida dubliniensis identification in Brazilian yeast stock collection. Mem Inst Oswaldo Cruz 98, 533-538.

Mensah TA, Tagoe SM (2018) Measurement of Yeast Growth Using Spectrophotometer. https://www.researchgate.net/publication/333667049

Montes de Oca R, Salem AZM, Kholif AE, Monroy H, Perez LS, Zamora LJ, Gutierez A (2016) Yeast: Description and structure. Yeast additive and animal production. Tamilnadu, PubBioMed Central Research Publishing Services,4-13. https://www.researchgate.net/publication/293605511

Qiangqiang Z, Jiajun W, LI L (1998) Storage of fungi using sterile distilled water or lyophilization: comparison after 12 years. Mycoses 41, 255- 257.

Ravimannan N (2016) Investigating alternative yeast storage methods. Int J Adv Res Biol Sci 3, 109-111. DOI:10.22192/ijarbs.

Suriasih K, Aryanta WR, Mahardika G, Astawa NM (2012) Microbiological and chemical properties of kefir made of Bali cattle milk. Food Sciand Quality Management 6, 1222. 
Tan GH, Mustapha N (2014) A comparative analy sis of preservation of functional food cultures by freeze- drying, liquid-drying and freezing methods. Direct Research J of Agriculture and Food Sci 2, 13-18.

Valente P, Gouveia FC, de Lemos GA, Pimentel D, van Elsas JD, Mendonca-Hagler LC, Hagler AN (1996) PCR amplification of the rDNA internal transcribed spacer region for differentiation of Saccharomyces cultures. FEMS Microbiol Lett 137, 253-256.

Widiastutik N, Alami NH (2013) Isolation and identification of yeast from Rhizosphere Rhizophora mucronata Wonorejo. J of Sci and Art Pomits 2, 1-5. 